\title{
Feasibility and Acceptability of Using Smartphone Apps in Diabetes Self-Management in an Underserved Population: Qualitative Study
}

Jenny Luo, PharmD; Shelley White-Means, PhD

University of Tennessee Health Science Center, Memphis, TN, United States

Corresponding Author:

Jenny Luo, PharmD

University of Tennessee Health Science Center

881 Madison

Memphis, TN,

United States

Phone: 4483521

Email: jluo2@uthsc.edu

\section{Abstract}

Background: The successful management of diabetes is a chronic endeavor. It involves a whole host of factors ranging from a consistent patient-provider relationship to regular physical activity. Not all patients with diabetes, however, have access to the resources needed for effective disease management. Health disparities contribute to a higher frequency of diabetes development in poor and minority populations. Moreover, health care disparities limit the care these patient populations receive. Because underserved populations have little to no access to traditional means of health care, providers must explore other avenues to reach this patient group. Mobile health (mHealth) has grown significantly in the last decade. With the fast-paced adoption of cell phones across all socioeconomic groups, mobile health presents the opportunity to offer patients a low-cost way to receive health information, to communicate with providers, and to self-manage chronic conditions. It has been well established that low-income, minority populations experience several barriers to receiving basic health care including uninsurance, limited transportation, and high out-of-pocket costs. The provision of health care via mobile devices may have the potential to address such health disparities. Little is known about the effectiveness of using mobile health and smartphone applications (apps) in underserved populations to help with diabetes management. Knowledge of these patients' interest in using mobile apps to augment their home self-management may have use in future implementations.

Objective: The objective of this study is to examine the perception of and willingness to use diabetes mobile health apps on smartphones in patients with limited access to primary care providers.

Methods: This study used purposive sampling to select patients for personal interviews. The study was conducted at a general hospital located in a part of town with predominate minority and low-income residency, as well as the highest diabetes prevalence rates. Semi-structured interviews were conducted according to McNamara's interview staging. A total of 15 interviews were collected and coded by the researcher according to the interpretative phenomenological analysis framework. An independent committee reviewed all interview transcripts and coding to verify trustworthiness of collection and analysis.

Results: The data produced 7 clusters related to smartphone app use and mHealth, each highlighting a component of the patient experience, which supported 3 overarching themes. The themes are as follows: despite limited knowledge about health apps and varying phone use patterns, patients were all willing to try at least one diabetes-related app; apps functions should be individualized to meet each patient's needs for maximum benefit; and barriers to app use were varied but commonly included knowledge and technological challenges and security issues.

Conclusions: Personal interviews of this underserved patient population demonstrated an interest in and willingness to try mobile health apps, despite limited knowledge about the technology. Responses indicate that tailoring app choices to individual needs, instead of choosing a multi-functional one-size-fits-all app, would provide the most benefit for at-home diabetes self-management. Smartphone apps may serve as a viable low-cost resource for patients with diabetes who have limited access to traditional health care providers.

(iproc 2018;4(2):e11792) doi: 10.2196/11792 
Edited by T Hale; this is a non-peer-reviewed article. Submitted 02.08.18; accepted 29.08.18; published 17.09.18.

Please cite as:

Luo J, White-Means $S$

Feasibility and Acceptability of Using Smartphone Apps in Diabetes Self-Management in an Underserved Population: Qualitative Study

iproc 2018;4(2):e11792

URL: http://www.iproc.org/2018/2/e11792/

doi: $\underline{10.2196 / 11792}$

PMID:

CJenny Luo, Shelley White-Means. Originally published in Iproceedings (http://www.iproc.org), 17.09.2018. This is an open-access article distributed under the terms of the Creative Commons Attribution License (https://creativecommons.org/licenses/by/4.0/), which permits unrestricted use, distribution, and reproduction in any medium, provided the original work, first published in Iproceedings, is properly cited. The complete bibliographic information, a link to the original publication on http://www.iproc.org/, as well as this copyright and license information must be included. 